Paricin, leider Substanzen, die bezüglich ihres optischen Verhaltens so gut wie unbekannt sind, aber schwerlich Einfluss auf die Ablenkung des Chinins laben; denn selbst wenn die beiden Alkaloide im reinsten Zustande optisch völlig unwirksam sein sollten, was noch nachzuweisen ist und somit die Linksdrehung des Chinins nicht beeinträchtigen würden, so bewirken sie doch andererseits, dass von den übrigen Basen, insbesondere von dem Conchinin, wenn solche mit Aether behandelt werden, ein nicht unerheblicher Theil mit in Lösung geht. Aber auch in dem Falle, dass beide Basen fehlten, nimmt Aether von Conchinin und Chinidin gerade so viel auf, dass sich die zweite Beobachtung, d. i. die mit dem angeblich unlöslichen Antheil, von der Wahrheit ziemlich entfernen muss. Dagegen wird sich der Chiningehalt nach Hesse wahrscheinlich in der Weise ermitteln lassen, dass man die neutralisirte schwefelsaure Lösung sämmtlicher Chinabasen mit einem kleinen Ueberschuss von weinsaurem Kalinatron ausfällt und den aus Chinin- and Chinidintartrat bestehenden Niederschlag nach vorheriger Auflösung in -verdünnter Schwefelsäure auf seine Ablenkung prüft, da anzunehmen ist, dass die grosse Differenz, die beide Basen für sich in Betreff ron a zeigen, auch bei ibren Tartraten entsprechend stattfinden wird.

\title{
Ueber eine neue Prüfungsmethode des Opiums auf seinen Morphin-
} gehalt. Als Grundlage fün diese Methode diente W. Stein*) die bekannte Eigenschaft des Morphiums aus der Jodsäure das Jod abzuscheiden, in Verbindung mit der Färbung, welche letzteres dem Chloroform ertheilt. Macht man eine reine Morphinlösung von bestimmtem Gehalte und verdümnt diese nach und nach mit bestimmten Wassermengen, so gelangt man endlich zu einem Verdünnungsgrade, bei welchem das aus der Jodsäure frei gemachte Jod das Chloroform so wenig färbt, dass man die Färbung nur noch mit Mühe erkennen kann; das ist die Empfindlichkeitsgrenze der Reaction. Stein fand für sein Auge dieselbe bei 1 Morphin in 20,000 Th. Wasser. In Betracht kommt, dass Alkohol, Essigsäure, Salzsäure, Ammon und fixe Alkalien sowie Erwärmung das Erscheinen der Farben verhindern oder abschwächen. Erwärmung vor dem Zusatz des Chloroforms beschleunigt dagegen die Reaction. Vor dem Zusatze des Chloroforms lässt man dann die Flüssigkeit wieder abkühlen. Will man nicht erwärmen, so muss man wenigstens $1 / 2$ Stunde stehen lassen, ehe man Chloroform zusetzt,

*) Archir d. Pharm. Bd. 198, p. 150. 
oder doch wheilt. Das Schutteln mit JChloroform muss ofter in Zwischenräumen von ca. 5 Winuten wiederholt werden. Ene verdinnte Schwefelsäure von $1: 4$ äusserte keine nachtheilige Wirkung; freie Schwefelsäure ist sogar zur Beschleunigung der Jodsüurewirkung anzuwenden. Nach dem Angeführten ist klar, dass man den Morplingehalt einer gegebenen Lösung, wenn deren Gewicht, bekannt ist, finden kann, wenn man dieselbe mit bekannten Wassermengen so lange verdünnt, bis die Grenze der obigen Reaction erreicht ist. Man könnte aber auch eine Farbenscala mit Hülfe von reinem Morphium auf die oben angegebeae Weise in Form von in Glasröhren eingeschlossener Jodcbloroformfiussigkeit herstellen, wovon jeder Ton einem bestimmten Morphingehalt entsprăche and die mit einer gegebenen Lösung erhaltene Farbe vergleichen. Stein lält es jedoch für sicherer, das Verschwinden einer Farbe, als die Gleichheit zweier Farben zu beurtheilen. Zur Entfernung der übrigen im Opium enthaltenen Stoffe, weiche wie das Morphium aus Jodsäure Jod abscheiden, hat Stein Kupferoxydsalze zweckmässig gefunden und wendet daher die Methode in folgender Weise an: 0,1 Grm. Opiumpulver und ebensoviel oder das doppelte Gewicht schwefelsaures oder essigsaures Kupferoxyd werden in einem Kochkölbchen mit einigen Tropfen verdünter Schwefelsäure eben durchfeuchtet, dann mit 100 Grm. destillirtem Wasser bis zum Kochen erhitat und $1 / 4-1 / 2$ Stunde stehen gelassen. Nach dieser Zeit wird filtrirt. Von diesem Filtrat werden $6 \mathrm{CC}$. mit ca. 0,06 Grm. Jodsäure*) und 2-3 Tropfen reiner Schwefelsüure zasammengebracht and wenn die Jodsäure gelöst ist, 3 CC. alkoholfreies Chloroform zugegeben. Nach während $1 / 4$ Stunde mehrmals wiederholtem Schütteln wird endlich stehen gelassen, um nach erfolgter Scheidung der Flüssigkeitsschichten die Farbung des Chloroforms zu beurtheilen. Wenn das geprüte Opiam $10 \%$ Morphium enthalten hätte, so würde in der angewendeten Menge 0,01 Grm. vorhanden gewesen sein und dieses wäre bei Anwendung von $100 \mathrm{Grm}$. Wasser in $10,000 \mathrm{Th}$. des letzteren gelöst. $\mathrm{Da}$ dies die halbe von Stein noch erkennbare Verdünnung ist, so entspräche eine kaum bemerkbare. Färbung des Chlorforms einem Gehalte ron $5 \%$ Morphin. Ist die Färbung dagegen sehr deutlich, so werden" $3 \mathrm{CC}$. des genannten Filtrats mit $3 \mathrm{CC}$. Wasser gemischt und ebenso behandelt,

*) Eine der Morphinmenge gleichè Menge Jodsäure ist genügend. Ein Ueberschuss schadet zwar nicht, doch kann sich bei gleichzeitig vorhandenem Ueberschusse von Kupfersalz jodsaures Kupferoxyd abscheiden. 
wie vorher. Zeigt sich nun die Grenzfürbung, so enthält das Opium 10\% Morphin; bleibt das Chloroform farblos, so liegt der Gehalt zwischen 5 und $10 \%$ und kann durch entsprechende Mischung des Filtrats mit Wasser noch näher ermittelt werden, wenn man sich nicht, wie es in den meisten Fällen beim Einkauf von Opium ausreichend ist, begnügt zu wissen, dass $10 \%$ Morphin eben nicht vorlanden sind.

Gänzlich unabhängig von Stein's Arbeit habe ich ebenfalls vor einigen Wocheil die Zersetzung von Jodsäure durch Morphin zur quantitativen Bestimmung des letzteren benutzt. Es handelte sich um die Entscheidung, ob ein Morphinpulver 0,005 Grm. oder 0,01 Grm. essigsaures Morphin enthalte. Ich operirte nalıezt in derselben Weise_wie Stein, benutzte jedoch anstatt Chloroform Schwefelkohlenstoff und bestimmte das frei gewordene von dem Schwefelkohlenstoff aufgenommene Jod, nach Entfernung der freien Säure durch Waschen, mit einer titrirten Lösang von unterschwefligsaurem Natron, führte also die Morphinbestimmung auf eine Jodtitrirung zurück. (Fresenius quantit. Analyse, 5. Aufl., pag. 541., d. «.) Tch erhielt im Ganzen sehr befriedigende-Resultate und werde darüber, sobald ich die Fehlerquellen etc. genauer erforscht habe, ausführlich berichten. (N.)

Ueber die Säurebestimmung im Rothwein. Die Säurebestimmung im Rothwein führt man nach F. Schwackhöfer*) am besten in der Art aus, dass man den Wein mit einer nach der Gottlieb'schen Methode bereiteten Lackmustinktur versetzt, alsdann mit Aézzbarytlösung übersättigt und den Barytüberschuss mittelst Schwefelsäure zurücktitrirt. Sobald der Sättigungspunkt nahe rückt, erzeugt jeder Tropfen Schwefelsäure an der Stelle, wo er in die Flüssigkeit einfällt, eine rothe Zone, die durch den gleichzeitig ansfallenden schwefelsauren Baryt sehr scharf hervorgehoben wird, und hat man in dem schnelleren und langsameren Verschwinden der rothen Farbe ein Wahrzeichen des herannahenden Sättigungspunktes. Ist derselbe fast erreicht, so genügen 2-3 Tropfen einer sehr verdünnten Schwefelsäure, um den Farbenübergang deutlich wahrzunehmen. Diese Methode kann natürlich auch bei Titrirungen des rohen Holzessigs und anderer dunkelgefärbter Flüssigkeiten in Anwendung gebracht werden. Zur Titrirung wurden je $10 \mathrm{CC}$. Rothwein

*) Annal. d. Oenologie Bd. 2 p. 347. 